\title{
GAMYB modulates $b H L H 142$ and is homeostatically regulated by TDR during rice anther tapetal and pollen development
}

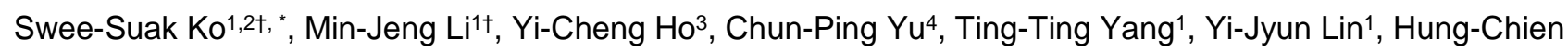
Hsing ${ }^{1}$, Tien-Kuan Chen ${ }^{1}$, Chung-Min Jhong ${ }^{1}$, Wen-Hsiung $\mathrm{Li}^{4,5}$, and Maurice Sun-Ben $\mathrm{Ku}^{3,6}$

${ }^{1}$ Academia Sinica Biotechnology Center in Southern Taiwan, 741 Tainan, Taiwan 


\section{Running title: TDR inhibits GAMYB modulating bHLH142 expression}

\section{Highlight:}

26 GAMYB can directly modulate the transactivation of the $b H L H 142$, but the modulation is repressed by TDR to 27 keep the homeostasis of $b H L H 142$ gene expression to ensure normal pollen development.

\section{Abstract}

30 GAMYB, UDT1, TIP2/bHLH142, TDR, and EAT1/DTD are important transcription factors (TFs) that play a 31 crucial role during rice pollen development. This study demonstrates that bHLH142 acts downstream of UDT1 32 and GAMYB and works as a "hub" in these two pollen pathways. We show that GAMYB modulates bHLH142 33 expression through specific binding to the MYB motif of bHLH142 promoter during early stage of pollen 34 development; while TDR acts as a transcriptional repressor of the GAMYB modulation of $b H L H 142$ by binding 35 to the E-box close to the MYB motif on the promoter. The up- and down-regulation of TFs highlights the 36 importance that a tight, precise, and coordinated regulation among these TFs is essential for normal pollen 37 development. Most notably, this study illustrates the regulatory pathways of GAMYB and UDT1 that rely on 38 bHLH142 in a direct and an indirect manner, respectively, and function in different tissues with distinct biological 39 functions during pollen development. This study advances our understanding of the molecular mechanisms of 40 rice pollen development.

42 Keywords: Oryza sativa, tapetum, pollen, bHLH142, UDT1, GAMYB, TDR, RNA FISH, DISH. 


\section{Introduction}

46 Pollen development during reproductive growth is a precise and complex process, affecting crop fertility and productivity. Therefore, gaining knowledge about the regulatory network of pollen development is critical for laying the groundwork for controlling pollen fertility in rice and production of hybrid rice. The tapetum layer is the innermost layer of the anther wall that provides nutrients for the development of microspores (Goldberg et al., 1993). Timely control of tapetal program cell death (PCD) is crucial for pollen maturation (Ko et al., 2017). Several transcription factors (TFs) are associated with tapetal PCD, including UNDEVELOPED TAPETUM1 (UDT1, bHLH164) (Jung et al., 2005), GIBBERELLIN MYB GENE (GAMYB) (Aya et al., 2009), TDR INTERACTING PROTEIN2(TIP2, bHLH142) (Fu et al., 2014; Ko et al., 2014), TAPETUM DEGENERATION RETARDATION (TDR, bHLH5) (Li et al., 2006), and ETERNAL TAPETUM 1/DELAYED TAPETUM DEGENERATION (EAT1/ DTD, bHLH141) (Ji et al., 2013; Niu et al., 2013). GAMYB is involved in Gibberellinregulated gene expression in anthers (Aya et al., 2009). EAT1/DTD regulates the gene expression of two aspartic proteases, $A P 25$ and $A P 37$, to execute tapetal PCD, and OsC4, OsC6, and RTS to maintain pollen wall development (Niu et al., 2013; Ji et al., 2013). Another TF PERSISTANT TAPETAL CELL1 (PTC1) also controls PCD and pollen wall development in rice (Li et al., 2011).

Lipid biosynthesis is important for the formation of pollen cell wall exines and Ubisch bodies in anthers (Huysmans et al., 1998). It has been reported that CYP703A3, a cytochrome P450 fatty acid hydroxylase, is an essential enzyme in sporopollenin biosynthesis, and its expression is regulated by GAMYB (Aya et al., 2009). CYP703A3 acts downstream of bHLH142 in the regulatory hierarchy (Aya et al., 2011; Ko et al., 2014). In addition, OsC6 (LTPL68) and OsC4 (LTP44) coding for lipid transfer protein precursors are also involved in the synthesis of pollen exines during anther development (Tsuchiya et al., 1994; Zhang et al., 2010). Consistently, YYI (LTP45) and Fatty acyl-CoA reductase (MS2), are downregulated in both ms142 and ptc1 mutants (Ko et al., 2014; Li et al., 2011). Rice polyketide synthase family protein PKS1 (YY2) is also known to function in sporopollenin metabolism (Wang et al., 2013; Zou et al., 2017). These proteins are essential for pollen maturation. During pollen development immature pollen grains are produced from microspores after meiosis. Meiotic recombination is important to exchange genetic information and create genetic diversity (Li et al., 2005). RAD51 plays an important role in homologous recombination during meiotic process (Shinohara et al., 1992).

It has been reported that GAMYB functions in parallel with UDT1 in rice to regulate early anther development (Liu et al., 2010). TDR transcript is decreased in gamyb-2 mutant, indicating that TDR functions downstream of GAMYB (Aya et al., 2009; Liu et al., 2010). Our previous gene hierarchy analysis suggested that bHLH142 acts downstream of GAMYB and UDT1 but upstream of EAT1 in rice pollen development (Ko et al., 
modulate the expression of EAT1 expression (Ko et al., 2014). Moreover, overexpression of homologous $b H L H 142$ leads to premature upregulation of EAT1 transcription and causes early tapetal PCD, suggesting that timely and precise control of bHLH142 level is critical to normal rice pollen development (Ko et al., 2017).

Past studies have extended our knowledge of the complex molecular regulatory cascades during pollen development. However, the interactions among the regulatory TFs identified so far and their positions in the regulatory hierarchy remain unclear. The present study extends our understanding of the underlying mechanisms of the interactions between GAMYB, TDR and bHLH142 during rice pollen development. We provide strong evidences from mutagenesis analysis, transient promoter assay (TPA), electrophoresis mobility shift assay (EMSA), and molecular dynamic simulation of TF-promoter interaction to support our findings.

\section{Materials and methods}

\section{Plant materials and growth conditions}

Rice (Oryza sativa) seeds of the ms142 and udt1 mutants in TNG67 background were obtained from the TRIM library (http:// trim.sinica.edu.tw/). Mutants of gamyb-2 (in Nipponbare background) and eat1 (in Hitomebore background) were requested from the National Institute for Agrobiological Sciences (NIAS), Japan. Mutant of $t d r$ was obtained from POSTECH Pohang, Korea. Rice seeds of mutants and their wild-type were sown in vermiculite for 3 weeks and then transplanted into soil in the Academia Sinica-Biotechnology Center in Southern Taiwan greenhouse for genetically modified organisms, Tainan, Taiwan.

\section{Rice transformation}

98 Constructs of the GAMYB (Os01g0812000) and TDR (Os02g0120500) full-length cDNA were PCR amplified using primer sets of GAMYB-BamHI-F and GAMYB-Sal-R, TDR-BamHI-F and TDR-Sall-R, respectively (Table S1). The PCR product is a $1688 \mathrm{bp}$ and $1671 \mathrm{bp}$ fragment, respectively. The fragment was digested with BamHI and ligated into pCAMBIA1390 vector containing the maize ubiquitin promoter. Expression of the selection marker Hptll gene that encodes hygromycin phosphotransferase was driven by cauliflower mosaic virus (CaMV) $35 S$ promoter. All constructs were confirmed by DNA sequencing. The plasmids were separately transformed and selected by antibiotic. Agrobacterium tumefaciens strain EHA105 was used to transfect TNG67 calli as described previously (Chan et al., 1993).

\section{Total RNA isolation and qRT-PCR}


Total RNA was isolated from rice tissues using TRIzol Plus RNA purification kit (Invitrogen) as described by the supplier. The stages of anthers were classified into the two stages according to spikelet length: meiosis (4 mm) and young microspore (YM, $6 \mathrm{~mm}$ ). Total RNA was treated with DNase (Promega), then $1 \mu \mathrm{g}$ RNA was used to synthesize the oligo(dT) primed first-strand cDNA using the M-MLV reverse transcriptase cDNA synthesis kit (Promega). Quantitative RT-PCR was performed using a CFX96 Real-Time PCR detection system (Bio-Rad, USA). Quantification analysis was performed using CFX Manager Software (Bio-Rad, USA). Primers used for qRT-PCR are listed in the Table S1. Ubiquitin-like 5 (Os01g0328400) was used as an internal control for normalization of gene expression levels. Each sample had three biological repeats.

\section{Transient promoter assay}

118 Transient promoter assay (TPA) was performed as previously described (Ko et al., 2014) with some modifications. In brief, protoplasts were isolated from young leaf tissue of 10-d-old rice seedlings. The reporter plasmid contained the CaMV35S minimal promoter and the $b H L H 142$ promoter $(3 \mathrm{~Kb})$ fused to the firefly luciferase gene (LUC). In the effector plasmids, GAMYB and TDR were under the control of the CaMV35S promoter. The pBI221 vector containing a CaMV35S promoter driving the expression of GUS was used as an internal control. To verify whether TDR might repress GAMYB modulation on the bHLH142 promoter, different molar ratios of TDR (1x,5x, and 10x) and GAMYB proteins were used in the TPA.

\section{Electrophoretic mobility shift assay}

Rice GAMYB and TDR proteins were expressed as a Maltose binding protein (MBP) fusion in E. coli. A total of

$25 \mu \mathrm{g}$ protein was incubated with the appropriate Dig-labelled DNA probes. Two fragment of the $b H L H 142$ probes were synthesized. Probe 1 contained a 66-mer oligonucleotide (-59 to $-124 \mathrm{bp}$ from the TSS) was synthesized (Table S1). It contains the MYB-like binding motif 5'-CAACAAA-3', at -103 to -109 bp from the TSS of $b H L H 142$, and an E-box on the bHLH142 promoter at $-73 \mathrm{bp}$ to $-78 \mathrm{bp}$ from the TSS of $b H L H 142$. Probe 2 from -977 to -1034 bp TSS, consisted of GARE and E-box, was synthesized (Table S1).

Prediction of 3D protein structures of transcription factors and their interaction with promoters by molecular dynamics simulation

The 3D protein structures of TDR, GAMYB and bHLH142 were constructed by MD simulation using ab initio modeling methods. MD simulation was performed by the NAMD (Nelson et al., 1996) program using parameters adopted from the CHARMM force field (Brooks et al., 1983). The full-length protein sequences of GAMYB (BAF06506.1), and TDR (NP_001045710.1) were obtained from the NCBI Information database. The DNA probes for binding to the TF proteins were generated by 3D-DART server (van Dijk and Bonvin, 2009). The 
models were minimized by removing unfavorable contacts, brought to $310 \mathrm{~K}$ by velocity rescaling, and equilibrated for $1 \mathrm{~ns}$. Before any MD trajectory was run, 40 ps of energy minimization was performed to relax the conformational and structural tensions. The minimum structure was the starting point for MD simulation. For this purpose, the protein molecule was embedded into a water simulation box and a cutoff distance of $12 \AA$ was employed for the non-bonded and electrostatic interactions. The heating process was performed from 0 to $310 \mathrm{~K}$ through Langevin damping with a coefficient of 10 ps21. A time step of 2 fs was employed for rescaling the temperature. After $20 \mathrm{ps}$ of heating to $310 \mathrm{~K}$, equilibration trajectories of $2 \mu \mathrm{s}$ were recorded, which provided the data for the structural and thermodynamic evaluations. The equations of motion were integrated with the Shake algorithm with a time step of $1 \mathrm{fs}$. Fig. 4 displays atomistic pictures of molecules generated using UCSF Chimera (Pettersen et al., 2004).

\section{RNA Fluorescent in situ Hybridization (FISH)}

For synthesis of bHLH142 probe, gene specific primer sets of 5'-CATGTTCAACACCAAGA TTCATTCG-3' and 5'- TGCAAACCATGACATACCAAAGATC-3' (Table S1), the same probe sequences as in our previous study(Ko et al., 2014), were used. We used DIG RNA labelling kit (SP6/T7) (Cat. No. 11175025910, Roche) to synthesize probes according to the manufacturer's instructions. FISH was performed, according to previous protocols(Jandura et al., 2017) with some modifications. Briefly, primary anti-DIG-POD, Fab fragment (Cat. no. 11207733910, Roche), and tyramide signal amplification (TSA) of the TSA Plus Cyanine 5 (Cy5) detection kit (NEL745001KT, PerkinElmer) were used for a single color FISH experiment.

Anthers at the early meiosis stage of TNG67 and homozygous mutant lines (ms142, gamyb-2, and udt1) were collected, fixed, dehydrated, embedded, tissue section to $10 \mu \mathrm{m}$, and performed in situ hybridization. Tissue samples were dewaxed, re-hydrated, protease (Cat. no. 03115836001, Roche) digested, glycine treated (UR-GLY001, Sigma-Aldrich), post-fixed, acetylated with acetic anhydride in triethanolamine, and then dehydrated. Slides were prehybridized at $37^{\circ} \mathrm{C}$ for $1 \mathrm{~h}$, hybridized with a probe at $58^{\circ} \mathrm{C}$ overnight, blocking was performed, incubated with anti-DIG-POD antibody at $37^{\circ} \mathrm{C}$ for $2 \mathrm{~h}$, and then washed three times (15 min each). After that, tissues were reacted with TSA working solution containing Cy5 Plus Amplification Reagent (300x dilution) at room temperature for 5 minutes, and washed twice. Afterwards, slides were counterstained with 4', 6-diamidino-2-phenylindole (DAPI) (Cat. no. D9542, Sigma-Aldrich). Florescence signals were observed using a Zeiss LSM710 confocal microscope equipped with a T-PMT under a Cy5 filter at excitation wavelength of 633 $\mathrm{nm}$ and emission wavelength of $670 \mathrm{~nm}$ onwards, and the DAPI signal was excited by a UV filter at a wavelength of $405 \mathrm{~nm}$.

\section{Two-Color FISH (DISH)}

The DISH technique(Brend and Holley, 2009) was used to show the co-expression of GAMYB and bHLH142 transcripts, as well as UDT1 and $b H L H 142$ transcripts in the same tissue of TNG67 anther at the early meiosis 
stage. To synthesize the GAMYB probe, gene specific primer sets of 5'- CGAGCTGGGAGGAGCAAAGGA-3' and 5'- TCCATGGCCCCTTCTTCAGC -3' were used. To synthesize the UDT1 probe, gene specific primer sets of 5'-GAGGAGGTGAAGGTGGAGGATGA-3' and 5'-CGCGAAGATGTTG CCGTTGA-3' were used. DIG RNA labelling Kit and Fluorescein (FITC) RNA Labeling Mix (Cat. no. 11685619910, Roche) were used to synthesize probes according to the manufacturer's instructions. Probes of $b H L H 142$ and GAMYB were simultaneously hybridized with tissue slices overnight at $58^{\circ} \mathrm{C}$. Then, anti-FITC-POD was conjugated with GAMYB probe overnight at room temperature, and then tissues were reacted with TSA working solution containing TSA Plus Cyanine 3 (Су3) (NEL744001KT, PerkinElmer) (at 1:100 dilution) at room temperature for 5 minutes, and then washed twice. Afterwards, tissues ware treated with $3 \%$ hydrogen peroxide at room temperature for 10 minutes to inactivate the first peroxidase of anti-FITC-POD. After that, anti-DIG-POD was conjugated with $b H L H 142$ probe at $37^{\circ} \mathrm{C}$ for $2 \mathrm{hr}$. Tissues were then stained with TSA working solution containing Cy 5 amplification reagent (at 1:300 dilution) at room temperature for $5 \mathrm{~min}$, and washed twice. For DISH experiment to study coexpression of $b H L H 142$ and UDT1, probes of $b H L H 142$ and UDT1 were hybridized with tissue slices overnight at $58^{\circ} \mathrm{C}$. Anti-FITC-POD, Fab fragment was conjugated with UDT1 probe overnight at room temperature. Then anti-DIG-POD was conjugated with $b H L H 142$ probe at $37^{\circ} \mathrm{C}$ for $2 \mathrm{hr}$. We also performed dye-swap experiments, reciprocal staining of TSA Plus Cy3 and Cy5 to confirm the consistency of RNA fluorescent signals. Fluorescent signals were observed under Zeiss LSM710 confocal microscope equipped with a T-PMT, using Cy3 or Cy5 filter at excitation and emission wavelengths of $543 \mathrm{~nm} / 600 \mathrm{~nm}$ or $633 \mathrm{~nm} / 670 \mathrm{~nm}$.

\section{Results}

\section{bHLH142 is a "hub" of the GAMYB- and UDT1-mediated pathways}

We studied the expression patterns of UDT1, GAMYB, TDR, bHLH142, and EAT1 in the anthers of the rice cultivar TNG67 at the meiosis and young microspore (YM) stages and also the expression pattern of bHLH142 in mutants defective in one of these TFs using qRT-PCR. Our results indicated that in anthers, except for EAT1, the other four TFs were expressed highly at the meiosis stage. Interestingly, bHLH142 was downregulated, while TDR and EAT1 were upregulated at the young microspore (YM) stage (Fig. 1A). The up- and downregulation of these TFs during anthers development indicate that precise control of the expression level of these TFs at different developmental stages is critical to normal rice pollen development. Several studies have reported that bHLH142 is located downstream of GAMYB and UDT1 (Fu et al., 2014; Ko et al., 2014). In this study, we investigated bHLH142 expression patterns in the anther of gamyb-2, udt1, and ms142 mutants at the meiosis stage. Our qRT-PCR data confirmed that $m s 142$ is a null mutant and does not express $b H L H 142$ mRNA in the anther. However, compared to the wild-type (WT), the expression of $b H L H 142$ was suppressed down to 
208 0.38-fold and 0.35-fold in udt1 and gamyb-2 mutants, respectively (Fig. 1B). These data suggest that when UDT1 is knocked out, the GAMYB-pathway is only partially functional with a reduced expression level of bHLH142. Similarly, when GAMYB is knocked out, the UDT1 pathway is still functional and some level of bHLH142 is retained in the anther of gamyb-2 (Fig. 1B). Taken together, these data suggest that the gene regulatory hierarchy of bHLH142 is located downstream of GAMYB-and UDT1-dependent regulatory pathways and serves as the "hub" of both pathways during rice pollen development.

To test the function of GAMYB, we generated GAMYB overexpression lines in the TNG67 background (denoted OE-GAMYB) and observed the male sterile phenotype in transgenic rice. OE-GAMYB exhibited light yellowish and smaller anthers than the WT. Moreover, pollen grain number was reduced and infertile; consequently, there was no seed developed in the OE-GAMYB transgenic lines (Fig. S1). The expression of bHLH142 was downregulated in the anther of gamyb-2 mutant, but upregulated in the anther of OE-GAMYB transgenic line at the meiosis stage (Fig. 1C). Clearly, loss of function and gain of function in GAMYB altered the expression of $b H L H 142$.

\section{Overexpressing TDR inhibits $b H L H 142$ gene expression}

We also generated transgenic rice overexpressing TDR, as driven by maize ubiquitin promoter (denoted OE$T D R$ ) for detailed characterization. Similar to OE-GAMYB, OE-TDR was male sterile and its anthers were small, white yellowish (Fig. 1D), and there was no pollen or seed developed. Our qRT-PCR data indicated that $t d r$ knockout mutant had slightly reduced bHLH142 expression (0.7 x WT) (Fig. 1E). Surprisingly, OE-TDR significantly repressed the expression of bHLH142 (only $0.1 \times$ WT) (Fig. 1E). We further examined the expression levels of $b H L H 142$ and TDR in the anthers of 15 individual OE-TDR transgenic lines at the meiosis stage by qRT-PCR and found that in the anther of TNG67, bHLH142 is expressed at a higher level than TDR at the meiosis stage (Fig. 1F). In 12 out of 15 OE-TDR lines, TDR was upregulated whereas bHLH142 was downregulated at the meiosis stage, compared to the WT. Except for three OE-TDR lines (\#5, \#11, and \#12), transcription of TDR was not upregulated, presumably due to the co-suppression effect. It is noteworthy that these three lines showed significant upregulation of bHLH142 (Fig. 1F) and have normal anther development similar to the WT. Clearly, whenever OE-TDR transgenic lines showed an increased TDR mRNA, their bHLH142 transcripts were significantly decreased $\left(r=-0.701^{\star *}\right)$. The expression levels of $T D R$ and $b H L H 142$ were fitted to a negative binomial regression model of $y=1.57 x^{2}-3.13 x+1.47\left(R^{2}=0.82\right)$ (Fig. 1G). We also assayed the expression patterns of GAMYB and UDT1 in the anther of OE-TDR transgenic lines and found no correlation ( $r=-0.27$ ) (Fig. S2). Using stable transgenic rice overexpressing TDR, we demonstrated that the gene expression 
239 level of $T D R$ is negatively correlated with that of $b H L H 142$. Thus, we hypothesize that TDR acts as a repressor 240 in the transactivation of $b H L H 142$ by GAMYB.

Identifying DNA-binding motifs of GAMYB and TDR

243 Since the DNA-binding domains (DBDs) of rice GAMYB and TDR, which belong to the MYB and bHLH families, respectively, have not been determined yet, we searched the TF databases to infer their motifs. The known positional weight matrices (PWMs) were collected from databases, including JASPAR (Khan et al., 2018), CisBP (Weirauch et al., 2014), Plant Cistrome (O'Malley et al., 2016), and two papers (Franco-Zorrilla et al., 2014; Sullivan et al., 2014). The DBD domain sequences of MYB members with known PWMs and the DBD domain sequences of bHLH members with known PWMs in plants were collected. The DBD of GAMYB is most similar to that of AtMYB33 with an $81.6 \%$ sequence identity (Fig. 2A), which is higher than the $80 \%$ threshold of DBD MYB set by the Cis-BP database, so we assumed that the PWM of GAMYB is similar to that of AtMYB33. The DBD of TDR is most similar to that of AtbHLH13 with a 33.7\% sequence identity (Fig. 2A). This sequence identity is lower than the $60 \%$ threshold set by Cis-BP, but we also assumed that the PWM of TDR is similar to that of AtbHLH13. We then checked these two putative PWMs by evolutionary conservation and EMSA. For the evolutionary conservation test, we checked the occurrences of the putative motifs of GAMYB and TDR on the $1.5 \mathrm{~kb}$ promoter sequence of $b H L H 142$ from the transcription start site (TSS) in a species using FIMO (Grant et al., 2011) with p-value $<0.005$. We indeed found both putative motifs in the orthologous bHLH142 promoters in Leersar perrieri, Sorghum bicolor, and Zea mays, though no putative motif of TDR was found in Brachypodium distachyon (Fig. 2B). This evolutionary conservation suggests the functionality of the two putative motifs.

In vivo and in vitro binding assays of GAMYB and TDR to bHLH142 promoter

Based on our data in Fig $1 \mathrm{C}$ and 2, we speculated that GAMYB modulates bHLH142 transactivation. A transient promoter assay (TPA) indicated that GAMYB activates the expression of bHLH142pro-LUC 3.8-fold in the protoplast of rice (Fig. 3A). This experiment was repeated three times to confirm the observation. We then conducted EMSA assays to verify the binding of GAMYB to the core motif of MYB in vitro. Because the $1.5 \mathrm{~kb}$ promoter of bHLH142 has two regions consisting of putative GAMYB and TDR binding motifs, respectively (Fig. 2B), two probes were synthesized to perform EMSA assays: probe 1 was a 66 -bp sequence from -59 to -124 bp from TSS and probe 2 was a 58-bp sequence from -977 to -1034 bp from TSS. When labeled probe 1 was incubated with GAMYB-MBP protein, a retarded band was observed. In addition, the specificity was confirmed 
that TDR-MBP protein binds to the E-box close to the MYB box on the promoter of bHLH142 probe 1 (Fig. 3C).

Similarly, when probe 2 was incubated with the GAMYB and TDR protein, respectively, a retarded band was also observed (Fig S3A, B). These data clearly demonstrated that GAMYB and TDR bind to the MYB motif and E-box on the probe 1 and probe 2 of $b H L H 142$ promoter, respectively.

As a previous study indicated that HvGAMYB regulates hydrolase genes during barley seed development and seed germination in a GA-dependent manner (Gubler et al., 1999), we examined whether GAMYB modulation of $b H L H 142$ expression is also $\mathrm{GA}_{3}$ dependent. Our TPA data indicated that adding $\mathrm{GA}_{3}$ from 0 to $10 \mu \mathrm{M}$ did not affect the transactivation of bHLH142pro-LUC (Fig. S4A). Thus, GAMYB modulates bHLH142 during pollen development independent of $\mathrm{GA}_{3}$. Because bHLH142 acts downstream of GAMYB and UDT1 (Fig. 1B), we were curious whether UDT1 also modulates bHLH142 promoter activity in the same way as GAMYB. TPA assay showed that UDT1 protein cannot directly transactivate bHLH142 (Fig. S4B). As TDR is located downstream of the GAMYB regulatory pathway(Liu et al., 2010), we were also curious whether GAMYB can modulate TDR promoter. Again, our TPA assay indicated that GAMYB does not directly regulate TDR (Fig. S4C).

\section{TDR represses GAMYB-mediated transcriptional activation of $b H L H 142$}

As transgenic rice overexpressing TDR suppressed the expression of bHLH142 (Fig. 1E, F), we hypothesized that TDR inhibits or interferes with the binding of GAMYB on the promoter of $b H L H 142$. To verify this hypothesis, we carried out TPA using different molar ratios of TDR and GAMYB proteins. The results showed that GAMYB increased bHLH142pro-LUC activity by up to 6 -fold in the absence of TDR protein, as expected. However, with addition of 1-, 5- and 10- molar ratios of TDR protein in the assays, the activity of bHLH142pro-LUC decreased progressively to 4.5-, 2.2- and 1.3-fold in comparison to the control activity (Fig. 3D). We further performed EMSA experiment to show the competition between GAMYB and TDR TFs on the promoter of bHLH142. Results with probe 1 indicated that when adding GAMYB protein only produced the protein/DNA complex with lower molecular weight (as indicated by black arrow). Adding TDR only, formed larger protein/DNA complex (as indicated by red arrow) due to the fact that TDR forms dimer for binding to its promoter. Adding $0.1 \times$ TDR protein, showed strong binding of TDR/DNA complex and slightly inhibition of GAMYB binding on bHLH142. However, adding $>1 \times$ TDR strongly suppressed GAMYB binding on bHLH142 promoter (Fig. 3E). These data strongly suggest that TDR acts as a repressor by inhibiting GAMYB modulation on bHLH142. In short, both in vivo and in vitro experiments suggest that TDR binds to the bHLH142 promoter and represses the transactivation of $b H L H 142$ through GAMYB.

\section{Molecular dynamic simulation shows binding of GAMYB and TDR proteins to bHLH142 promoter}


To illustrate how GAMYB and TDR interact to regulate the transactivation of $b H L H 142$ by binding to its promoter,

Protein Modeling and Molecular Dynamic (MD) simulation were conducted to determine their protein-DNA interactions. The 3D structures of GAMYB and TDR, were obtained by ab initio protein modelling methods through MD simulation in an aqueous environment(Nelson et al., 1996). GAMYB, which belongs to the MYB family, is able to bind DNA as a monomer, presumably due to the involvement of an additional $\mathrm{N}$-terminal tail that wraps around the DNA and makes base contacts in the minor groove (Hosoda et al., 2002; Ogata et al., 1994). In contrast, TDR forms a homodimer in which the two monomers make identical contacts with the DNA. TDR contains a bHLH domain of 281-330 amino acids. The HLH region comprises two amphipathic $\alpha$-helices separated by a loop of variable length and sequence, allowing the formation of homodimers or heterodimers (Wei and Chen, 2018).

GAMYB and TDR proteins possess electrical and hydrophobic interactive surface patches for protein/DNA interaction. GAMYB protein is predicted to bind to the AGCAACAAACCACTCTTC sequence on the bHLH142 promoter (-94 to -111 bp from TSS) (Fig. 4A). Our modeling also demonstrated that TDR binds an E-box (CAGCTG) on the bHLH142 promoter at -73 to -78 bp from TSS (Fig. 4B). Normally, dissociation Gibbs free energy $(\Delta G)$ is used to measure the binding affinity. The binding free energies for the binding affinity of GAMYB to CAACAAA and the binding of TDR to CAGCTG on the $b H L H 142$ promoter were $-170 \mathrm{kcal} / \mathrm{mol}$ and -319 $\mathrm{kcal} / \mathrm{mol}$, respectively (Fig. 4C, D). We found that TDR dimer/DNA complex is more stable than GAMYB/DNA complex. Thus, whenever the GAMYB protein level is high, it will transactivate bHLH142 gene expression; however, whenever the TDR protein level is high, it acts as a repressor because it has a higher binding affinity for more stable complex formation.

\section{Spatial expression patterns of $b H L H 142$}

325 We used RNA fluorescence in situ hybridization (FISH) for direct visualization of the spatiotemporal expression pattern of bHLH142 (Huang et al., 2019; Javelle et al., 2011). A strong RNA FISH signal was observed in anthers (Fig. 5A); red strong fluorescence signals of bHLH142 transcript (as indicated by arrows) were detected in the tapetal layer, middle layer, and meiocytes, with some weak signals detected in the epidermal outer layer and the endothecium of the anther of the WT. In contrast, no apparent bHLH142 signal was detected in the anther of $m s 142$. In the anthers of $u d t 1$ mutant only weak signals of bHLH142 mRNA, presumably from the GAMYBdependent pathway, were seen in the tapetal layer but not in the meiocytes. In the anther of gamyb-2, some bHLH142 FISH signals were detected in the meiocytes and in the tapetal cells (Fig. 5A). Taken together, these data suggest that FISH signals in the anther of gamyb-2 mutant mimic the UDT1-dependent regulatory pathway that regulates $b H L H 142$ in both tapetal layer (sporophyte) and meiocyte (gametophyte). However, the udt1 
detection of the same target genes using Dig-labelled probe and NBT/BCIP detection of the same paraffin blocks of FISH also showed an identical ISH and FISH signal for each mutant as well as the WT (Fig. S5). Differential interference contrast microscopy images of the anther morphology showed that the anther of $u d t 1$ exhibited degenerated meiocytes at the early stage of meiosis (Fig. S6).

Moreover, our two-color fluorescence in situ hybridization (DISH) demonstrated that both GAMYB and bHLH142 genes co-expressed in the same tissue slice of WT anther at the early meiosis stage. Green spots indicate the fluorescence signal of GAMYB mRNA expressed in the anther walls and meiocytes (Fig. 5B). Red spots indicate $b H L H 142$ expressed in the tapetal layer and partly in the meiocytes (Fig. 5B). Co-expression of GAMYB and bHLH142 mRNAs was found only in the tapetal cells (Fig. 5B yellow spots). These data reveal that GAMYB regulates bHLH142 transcript specifically in the tapetal layer. Dye-swap of the two probes showed similar fluorescence signals (Fig. S7). In addition, we performed DISH to detect the expression of both UDT1 and $b H L H 142$ in anther, which suggests the co-expression of these two genes in tapetal cells and meiocytes (Fig. 5C). Taken together, the analyses of RNA FISH using gamyb-2 and udt1 mutants and DISH with TNG67 demonstrated that $b H L H 142$ mRNA is indeed differentially expressed in different tissues. We hypothesize that GAMYB and UDT1 TFs regulate $b H L H 142$ and might have unique biological functions in these two pollen developmental pathways.

\section{Expression and putative functions of $b H L H 142$ in the GAMYB- and UDT1-dependent regulatory}

\section{pathways}

Next, we analyzed the expression of some known pollen marker genes that are significantly downregulated in ms142 and examined whether their expressions are altered in the anthers of gamyb-2 and udt1 mutants, respectively. Interestingly, some marker genes were downregulated in gamyb-2 and $m s 142$, but not significantly altered in udt1 mutant (named the GAMYB-dependent pathway). On the other hand, some were downregulated in udt1 and ms142, but not significantly changed in gamyb-2 (named the UDT1-dependent pathway). Our qRTPCR analysis indicated that the transcripts for some marker genes associated with lipid transport, such as $Y Y 1$, OsC4, and LTP2, were sharply decreased in the anthers of ms 142 and gamyb-2 mutants but not in that of udt 1 (Fig. 6A). However, RAD51, a marker gene involved in meiotic homologous recombination (Shinohara et al., 1992), was downregulated in both $u d t 1$ and $m s 142$, but not in gamyb-2 (Fig. 6B). Also, PTC1 and EAT1 were greatly downregulated in udt1 and ms142, but not in gamyb-2 (Fig. 6B). The expression of some other genes, such as TDR, MYB80, sporopollenin biosynthesis genes (CYP703A3 and CYP704B2), and PKS1 (YY2), were simultaneously downregulated in these three mutants (Fig. 6C). Collectively, these results reveal that some known pollen markers acting downstream of bHLH142 are dependent on the UDT1- or GAMYB- pathway, while some others are dependent on both pathways during rice pollen development. 


\section{Discussion}

GAMYB, UDT1, bHLH142, and TDR are master transcriptional regulators of rice tapetal and pollen development at the meiosis and early microspore stages. Previous studies suggest that GAMYB and UDT1 work in two parallel pathways (Liu et al., 2010) and bHLH142 acts downstream of GAMYB and UDT1 (Ko et al., 2014). This study further demonstrates that bHLH142 acts as a "hub" of these two pathways. We showed that GAMYB directly modulates $b H L H 142$ transcription by binding to its promoter (Fig. 3A, B), but UDT1 does not directly transactivate bHLH142 expression (Fig. S4B). Presumably, UDT1 (bHLH164) needs to interact with another bHLH to form a heterodimer to co-modulate $b H L H 142$ expression. This is similar to our previous discovery that two bHLHs, bHLH142 and TDR (bHLH5), form a heterodimer to co-modulate another downstream bHLH gene, EAT1 (bHLH141) (Ko et al., 2014). Alternatively, there is an unknown TF acting downstream of UDT1 that might directly modulate bHLH142. In Arabidopsis, the two TFs MS188 (MYB80) and AMS (a bHLH, TDR homolog in rice) form a complex to activate the expression of the fatty acid hydrolase gene CYP703A3 for sporopollenin biosynthesis (Xiong et al., 2016). Clearly, spatial and temporal coordination among the regulatory TFs is essential for rice pollen maturation. Using mutagenesis, FISH and DISH, we demonstrated that GAMYB specifically modulates bHLH142 expression in the tapetal cells (Fig. 5). Our qRT-PCR data showed that YY1 (LTP45), LTP2, and OsC4 (LTP44) transcripts are downregulated in the anther of gamyb-2 and ms142, but not significantly altered in udt1 (Fig. 6A). These results suggest that the expression of these lipid transfer genes is dependent on bHLH142 via a GAMYB-dependent pathway. UDT1 modulates bHLH142 expression in the tapetal cells and meiocytes (Fig. 5), indicating that the UDT1-dependent pathway regulates bHLH142 for sporophytic and gametophytic development during rice pollen development (Fig. 5). RAD51 is downregulated in both $u d t 1$ and ms142 mutants (Fig. 6B) and might be associated with meiosis defective in $u d t 1$ and ms142 mutants. Two TFs, PTC1 and EAT1, are involved in UDT1-dependent pathway (Fig. 6B). In other words, the UDT1-dependent pathway indirectly modulates $b H L H 142$ in broad tissue compartments including sporophyte (tapetum) and gametophyte (meiocyte). This result is in agreement with the function of bHLH142 in tapetal layer differentiation(Fu et al., 2014) and its regulation of various metabolic pathways during rice pollen development (Ranjan et al., 2017).

This study also clearly demonstrated that TDR plays an important role in regulating the homeostatic gene expression of $b H L H 142$. Transgenic lines overexpressing TDR showed a differential TDR expression pattern and the TDR and bHLH142 transcript levels were negatively correlated (Fig. 1F, G). Our TPA demonstrated that a high amount of TDR protein can repress the transactivation of $b H L H 142$ expression by GAMYB (Fig. 3D). Moreover, EMSA assay suggests that TDR directly binds to the E-box adjacent to a MYB motif on the bHLH142 promoter region (Fig. $3 \mathrm{C}$ ), which in turn suppressed the transactivation of $b H L H 142$ expression by GAMYB in 
402

a competitive manner (Fig. 3E). Hence, based on this and previous studies, we propose that TDR has at least three molecular functions during rice pollen development. First, it controls homeostasis of $b H L H 142$ by inhibiting the transactivation of $b H L H 142$ by GAMYB (Fig. 3). Second, TDR interacts with bHLH142 to form a heterodimer (bHLH142/TDR) to co-modulate EAT1 expression (Ko et al., 2014), and a sharp upregulation of EAT1 mRNA is found at the YM stage (Fig. 1A). Moreover, overexpressing bHLH142 caused premature upregulation of EAT1(Ko et al., 2017). Third, TDR interacts with EAT1 (Ji et al., 2013; Ko et al., 2014; Niu et al., 2013) in regulating the expression of the downstream genes for pollen maturation.

Based on the results from this and others studies, we propose an updated regulatory model of rice pollen development (Fig. 7). First, bHLH142 acts downstream of UDT1 and GAMYB and works as a "hub" in these two pollen development pathways (Fig. 1B). Second, GAMYB, but not UDT1, can directly modulate the transactivation of $b H L H 142$ promoter (Fig. 3A, Fig. S2B). Third, GAMYB modulates $b H L H 142$ at the early stage of meiosis (Fig. 1A). Consistently, from the late meiosis to early YM stages, a high level of bHLH142 transcript accumulates in the cytosol of tapetum cells (Ko et al., 2014). Fourth, in the meantime, TDR regulates the homeostasis of $b H L H 142$ expression by binding to an E-box adjacent to the MYB motif on its promoter region and inhibits GAMYB's modulation of $b H L H 142$ (Fig. 3). At the YM stage, TDR interacts with bHLH142 to comodulate EAT1 promoter as reported previously (Ko et al., 2014). EAT1 in turn regulates AP37 to turn on tapetal PCD (Niu et al., 2013) at the YM stage. Finally, both FISH and DISH data reveal the modulation of bHLH142 by GAMYB in the tapetal layer (Fig. 5) and the GAMYB-dependent pathway functions in lipid transport (Fig. 6A). However, the UDT1-dependent pathway plays more important roles in meiosis and tapetal programmed cell death (Fig. 6B). Overall, the present study underlines the importance of a precise and coordinated expression of these regulatory TFs spatially and temporally for pollen development.

\section{Accession numbers}

Sequence data from this article can be found in the GenBank/EMBL database under the following accession numbers: bHLH142 (Os01g0293100), protein (NP_001042795.1); GAMYB (Os01g0812000); UDT1 (Os07g0549600), and TDR (Os02g0120500). Additional loci are presented in Table S1.

\section{Acknowledgements}

We would like to thank the TRIM, Tos17, and Postech mutant libraries for providing mutant seeds for this study. We thank the Academia Sinica Transgenic Plant Laboratory for transforming the OE-TDR rice. We thank the GMO greenhouse of the Biotechnology Center in Southern Taiwan (AS-BCST) for providing space and technical support. We thank the Confocal Microscope Core Facility of AS-BCST for confocal microscopy and imaging 
435 assistance. We thank the DNA Sequencing Core Facility of the Institute of Biomedical Sciences of Academia 436 Sinica for providing DNA sequencing services. We appreciate Miranda Loney for English editing. This work was 437 supported by grants of AS-BCST and partly by The Ministry of Science and Technology to S.S. Ko (MOST 107438 2313-B-001-005), and AS-109-TP-10, Academia Sinica to W.H. Li.

439

\section{Conflicts of interest}

441 The authors declare that there are no conflicts of interest.

\section{Author contributions}

444 S.-S.K. conceived and designed the research. M.-J.L. discovered bHLH142 promoter is directly modulated by 445 GAMYB. Y.-C. H. and C.-P. Y. performed MD simulation and TFBS analysis. M.-J.L., T.-T.Y., Y.-J.L., H.-C.H., 446 T.-K.C., and C.-M.J. carried out the experiments. S.-S.K., M.-J.L., Y.-C. H., C.-P. Y. analyzed and prepared the 447 data. M.S.-B.K. and W.-H.L. suggested on the experiments. S.-S.K., M.S.-B.K., Y.-C. H., and W.-H.L. wrote the 448 article. 
452 Fig. 1. Expression patterns of bHLH142 and expression relationship between bHLH142 and TDR. (A) 453 Expression patterns of UDT1, GAMYB, bHLH142, TDR, and EAT1 in anthers of TNG67 at the meiosis (Mei) and young microspore (YM) stages. (B) Expression patterns of bHLH142 in the anthers of udt1, gamyb-2, and ms142 mutants at the meiosis stage. (C) Down- and up-regulation of bHLH142 in gamyb-2 and OE-GAMYB (line \#6) anthers, respectively. (D) Comparison of the spikelet morphology between TNG67 (WT) and OE-TDR \#4 transgenic rice. The photo was taken one day before anthesis. Bar $=1 \mathrm{~mm}$. (E) Expression patterns of bHLH142 in the anthers of $t d r$ mutant (in Dongjin background) and OE-TDR (in TNG67 background) at the meiosis stage. qRT-PCR data are normalized with respective to WT. (F) Expression patterns of TDR and $b H L H 142$ in OE-TDR transgenic lines. RNA of anthers at the meiosis stage was collected from 15 independent OE-TDR transgenic lines. (G) Relationship between bHLH142 and TDR mRNA levels in TNG67 (WT) and 15 OE-TDR transgenic lines. Regression analysis fit to a binomial regression equation of $y=1.57 x^{2}-3.13 x+1.47$ $\left(R^{2}=0.82\right) ; n=16$ lines. Gene expression levels were normalized to Ubi5 using qRT-PCR. Error bars indicate the SD among three technical replicates. Mei, meiosis; VP, vacuolated pollen. Each experiment was repeated three times.

Fig. 2. Inferring DNA-binding motifs of $G A M Y B$ and $T D R$, and identifying their TF binding sites in the promoter sequence of bHLH142. (A) DNA-binding domain (DBD) sequences of GAMYB (top) and TDR (bottom) and their top 4 similar DBDs of TFs with known motifs are shown. The logo of the known motif of AtMYB33 (/AtbHLH13) that has the best similar score with GAMYB (/TDR) is shown at the right panel. The two known motifs (PWMs) are from the Plant Citrome and Cis-BP databases. (B) Alignment of promoter sequences of bHLH142 and four orthologues are shown where two regions in the promoters have been identified as putative TF binding sites of GAMYB and TDR, respectively. The binding site of GAMYB (/TDR) is enclosed by a red (/blue) box.

Fig. 3. In vivo and in vitro assays of binding of GAMYB and TDR to bHLH142 promoter. (A) Transactivation of the $b H L H 142$ promoter sequence $(3 \mathrm{~kb})$ fused to the firefly luciferase gene $(L U C)$ reporter gene by GAMYB in rice protoplasts. Different effectors were co-transfected with the reporter and internal control plasmid (pBI221). The data represent means of three independent transient transformations. Transient transformation without the effector plasmid (mini35p) was used as a negative control. Bars indicate the SD of three biological replicates. 480 The experiment was repeated three times. (B) EMSA assay of binding of GAMYB to the promoter region of bHLH142. Probe 1 at -59 to $-124 \mathrm{bp}$ from the transcription start site (TSS) of $b H L H 142$ promoter was synthesized. 
DNA complex (B,C). The EMSA experiments were repeated three times. (D) Promoter transient assay indicated that TDR protein inhibited GAMYB modulation on $b H L H 142$ promoter-LUC activity. Different molar ratios of TDR (1x, 5x, and 10x) and GAMYB proteins were used for transient assays. The experiment was repeated three times. Bars indicate the SD of three biological replicates. (E) EMSA data indicated that TDR inhibited GAMYB binding on $b H L H 142$ promoter probe 1. The black and red arrows indicated GAMYB/ DNA complex and TDR/ DNA complex, respectively.

Fig. 4. Molecular dynamics simulations of protein/DNA interaction between GAMYB monomer or TDR homodimer and bHLH142 DNA. (A) Binding of the predicted amino acid patches on GAMYB to the bHLH142 promoter sequence AGCAACAAACCACTCTTC (-94 to -111 bp from TSS). (B) Binding of the predicted amino acid patches on TDR homodimer to the bHLH142 promoter sequence CAGCTG (-73 to -78 bp from TSS). (C) Interaction between GAMYB and the CAACAAA motif with a binding energy of $-170 \pm 57 \mathrm{kcal} / \mathrm{mol}$. (D) Interaction of TDR with an E-box (CAGCTG) with a binding energy of $-319 \pm 54 \mathrm{kcal} / \mathrm{mol}$.

Fig. 5. RNA fluorescent in situ hybridization (FISH) study of the expression patterns of bHLH142 in mutants and two-color fluorescent in situ hybridization (DISH) study of the co-expression of UDT1 and GAMYB with bHLH142 in anthers at early meiosis. (A) RNA single color FISH showing the expression pattern of $b H L H 142$ in the anthers of WT, ms 142, udt1 and gamyb-2 mutants during early meiosis. Anther tissues were stained with TSA working solution containing Cyanine 5 Plus Amplification Reagent (300x dilution). Ex/Em: 633/670 nm. The arrow indicates the FISH signal (red color). Tissues were counter stained with DAPI (4',6-diamidino-2-phenylindole).

E, epidermis; En, endothecium; ML, middle layer; T, tapetum. Bars $=20 \mu \mathrm{m}$. (B) DISH showing coexpression of GAMYB and $b H L H 142$ in the tapetal layer of the anther. Green and red spots show fluorescent signals of GAMYB and bHLH142, respectively. Yellow spots show the co-expression of GAMYB and bHLH142 mRNAs in the tapetal cells (white arrows). (C) DISH showing co-expression of UDT1 and bHLH142 in the tapetal layer and meiocytes of the anther. Green and red spots show fluorescent signals of UDT1 and bHLH142, respectively Yellow spots show co-expression of UDT1 and bHLH142 in the tapetal cells (white arrows) and meiocytes (blue arrow heads). Pictures were taken under 100X objective oil immersion lens. T, tapetal layer; M, meiocytes. Bars $=20 \mu \mathrm{m}$.

513 Fig. 6. Dependence of the expression of pollen marker genes on GAMYB, UDT1, or both pathways. Expression patterns of some pollen marker genes in the anthers of $u d t 1$, gamyb-2, and ms 142 mutant lines were studied using qRT-PCR. (A) YY1, OsC4, and LTP2 were found dependent on GAMYB- and bHLH142-regulatory 
pathways. (B) RAD51, PTC1, and EAT1 were dependent on UDT1- and bHLH142-regulatory pathways. (C) TDR, CYP703A3, Cyp704B2, MYB80, and PKS1/YY2 were dependent on both pathways. Gene expression levels were normalized to Ubi5, and the fold change relative to the respective Wild-type is presented. Error bars indicate the SD of three technical replicates. Each experiment was repeated three times.

Fig. 7. Model of this study. TF of bHLH142 acts downstream of UDT1 and GAMYB and works as a "hub" in these two pollen pathways. GAMYB can directly modulate the transactivation of the $b H L H 142$ promoter, but UDT1 cannot. GAMYB modulates $b H L H 142$ at the early stage of meiosis, but the modulation is repressed by TDR at the young microspore stage to keep the homeostasis of $b H L H 142$ gene expression to ensure normal pollen development. Our FISH and DISH data reveals that GAMYB modulates bHLH142 in the tapetal layer and GAMYB-dependent pathway functions in lipid transport. However, the UDT1-dependent pathway regulates bHLH142 in the tapetal layer and meiocytes and plays several other roles in meiosis and tapetal programmed cell death. YM, young microspore stage; $M$, meiocytes; $T$, tapetum; PCD, programmed cell death.

\section{Supplementary data}

Table S1. Primers used in this study.

Fig. S1. Phenotype of the TNG67 wild-type and overexpressing GAMYB transgenic line.

(A) Construction map of overexpressing GAMYB driven by Ubiquitin promoter. (B) Genomic PCR confirmed insertion of Ubi::GAMYB in transgenic lines. (C) Plant phenotype at the seed maturation stage. (D) Spikelet at one day before anthesis. (E) Pollen stained with $1 \% \mathrm{I}_{2} / \mathrm{KI}$ solution. Left panel is $\mathrm{WT}$, right panel is OE-GAMYB \#6. Scale bars, $20 \mathrm{~cm}(C), 1 \mathrm{~mm}(\mathrm{D}), 50 \mathrm{um}(\mathrm{E})$.

Fig. S2. Gene expression level of GAMYB and UDT1 were not altered in the anther of OE-TDR transgenic lines. RNA of anthers at the meiosis stage was collected from the Wild-type (TNG67) and 15 independent OE-TDR transgenic lines. The Pearson correlation coefficient between GAMYB and UDT1 mRNA is -0.27 . Bars indicate $\mathrm{SD}$ of mean from three technical replicates. $n=16$ lines.

Fig. S3. EMSA results indicated GAMYB and TDR TFs bind to $b H L H 142$ promoter sequences at -977 to -1034 bp from TSS. (A) GAMYB protein bound to the promoter region of bHLH142. (B) TDR protein bound to the 
promoter was synthesized. Arrow indicated protein and DNA complex. The experiment was repeated three times.

Fig. S4. Transient promoter analysis. (A) GAMYB modulation of $b H L H 142$ promoter is independent of $\mathrm{GA}_{3}$. (B) UDT1 does not directly modulated by bHLH142pro-LUC activity. c, TDR promoter does not modulated by

Fig. S5. Traditional Dig-labelled antisense probe of bHLH142 in the anthers of WT, ms142, udt1 and gamyb-2 mutants at early meiosis stage. Transverse sections of anther were hybridized with antisense or sense Diglabeled probe of $b H L H 142$. Bars $=20 \mu \mathrm{m}$.

Fig. S6. DIC of the anthers of WT, ms142, udt1 and gamyb-2 mutants at the early meiosis stage for RNA FISH analyze bHLH142 mRNA expression patterns. The arrows indicates degenerated meiocytes in udt1 mutant. Bars $=20 \mu \mathrm{m}$.

Fig. S7. Two-color fluorescent ISH (DISH) of GAMYB and $b H L H 142$ mRNAs in the anther of TNG67 at the early

stage of meiosis. DISH experiment performed reciprocal staining of TSA Plus Cy3 and Cy5 to show RNA fluorescence signals. Fluorescence signals were observed under a Zeiss LSM710 confocal microscope equipped with a T-PMT, using Cy3 or Cy5 filter at excitation/emission wavelengths of 543/600 nm and 633/670 $\mathrm{nm}$, respectively. Pictures were taken under $40 \mathrm{X}$ objective lens. Bars $=20 \mu \mathrm{m}$.

\section{References}

Aya K, Suzuki G, Suwabe K, Hobo T, Takahashi H, Shiono K, Yano K, Tsutsumi N, Nakazono M, Nagamura Y, Matsuoka M, Watanabe M. 2011. Comprehensive network analysis of antherexpressed genes in rice by the combination of 33 laser microdissection and 143 spatiotemporal microarrays. PLoS One 6, e26162.

Aya K, Ueguchi-Tanaka M, Kondo M, Hamada K, Yano K, Nishimura M, Matsuoka M. 2009. Gibberellin modulates anther development in rice via the transcriptional regulation of GAMYB. Plant Cell 21, 1453-1472.

Brend T, Holley SA. 2009. Zebrafish whole mount high-resolution double fluorescent in situ hybridization. J Vis Exp.

Brooks BR, Bruccoleri RE, Olafson BD, States DJ, Swaminathan S, Karplus M. 1983. Charmm - a Program for Macromolecular Energy, Minimization, and Dynamics Calculations. Journal of computational chemistry 4, 187-217. 
Chan MT, Chang HH, Ho SL, Tong WF, Yu SM. 1993. Agrobacterium-mediated production of transgenic rice plants expressing a chimeric alpha-amylase promoter/beta-glucuronidase gene. Plant Mol Biol 22, 491-506.

Franco-Zorrilla JM, López-Vidriero I, Carrasco JL, Godoy M, Vera P, Solano R. 2014. DNAbinding specificities of plant transcription factors and their potential to define target genes. Proc Natl Acad Sci U S A 111, 2367-2372.

Fu Z, Yu J, Cheng X, Zong X, Xu J, Chen M, Li Z, Zhang D, Liang W. 2014. The rice Basic HelixLoop-Helix transcription factor TDR INTERACTING PROTEIN2 is a central switch in early anther development. Plant Cell 26, 1512-1524.

Goldberg RB, Beals TP, Sanders PM. 1993. Anther development: basic principles and practical applications. Plant Cell 5, 1217-1229.

Grant CE, Bailey TL, Noble WS. 2011. FIMO: scanning for occurrences of a given motif. Bioinformatics 27, 1017-1018.

Gubler F, Raventos D, Keys M, Watts R, Mundy J, Jacobsen JV. 1999. Target genes and regulatory domains of the GAMYB transcriptional activator in cereal aleurone. Plant J 17, 1-9. Hosoda K, Imamura A, Katoh E, Hatta T, Tachiki M, Yamada H, Mizuno T, Yamazaki T. 2002. Molecular structure of the GARP family of plant Myb-related DNA binding motifs of the Arabidopsis response regulators. Plant Cell 14, 2015-2029.

Huang K, Baldrich P, Meyers BC, Caplan JL. 2019. sRNA-FISH: versatile fluorescent in situ detection of small RNAs in plants. Plant J 98, 359-369.

Huysmans S, El-Ghazaly G, Smets E. 1998. Orbicules in angiosperms: Morphology, function, distribution, and relation with tapetum types. The Botanical Review 64, 240-272.

Jandura A, Hu J, Wilk R, Krause HM. 2017. High resolution fluorescent in situ hybridization in Drosophila embryos and tissues using tyramide signal amplification. J Vis Exp.

Javelle M, Marco CF, Timmermans M. 2011. In situ hybridization for the precise localization of transcripts in plants. J Vis Exp, e3328.

Ji C, Li H, Chen L, Xie M, Wang F, Chen Y, Liu YG. 2013. A novel rice bHLH transcription factor, DTD, acts coordinately with TDR in controlling tapetum function and pollen development. Mol Plant 6, 1715-1718.

Jung KH, Han MJ, Lee YS, Kim YW, Hwang I, Kim MJ, Kim YK, Nahm BH, An G. 2005. Rice Undeveloped Tapetum1 is a major regulator of early tapetum development. Plant Cell 17, 27052722.

Khan A, Fornes O, Stigliani A, Gheorghe M, Castro-Mondragon JA, van der Lee R, Bessy A, Chèneby J, Kulkarni SR, Tan G, Baranasic D, Arenillas DJ, Sandelin A, Vandepoele K, Lenhard B, Ballester B, Wasserman WW, Parcy F, Mathelier A. 2018. JASPAR 2018: update of the open-access database of transcription factor binding profiles and its web framework. Nucleic Acids Res 46, D260-d266. 
Ko SS, Li MJ, Ku MS-B, Ho YC, Lin YJ, Chuang MH, Hsing HX, Lien YC, Yang HT, Chang HC, Chan MT. 2014. The bHLH142 transcription factor coordinates with TDR1 to modulate the expression of EAT1 and regulate pollen development in rice. Plant Cell 26, 2486-2504.

Ko SS, Li MJ, Lin YJ, Hsing HX, Yang TT, Chen TK, Jhong CM, Ku MS. 2017. Tightly controlled expression of bHLH142 is essential for timely tapetal programmed cell death and pollen development in rice. Front Plant Sci 8, 1258.

Li H, Yuan Z, Vizcay-Barrena G, Yang C, Liang W, Zong J, Wilson ZA, Zhang D. 2011. PERSISTENT TAPETAL CELL1 encodes a PHD-finger protein that is required for tapetal cell death and pollen development in rice. Plant Physiol 156, 615-630.

Li N, Zhang DS, Liu HS, Yin CS, Li XX, Liang WQ, Yuan Z, Xu B, Chu HW, Wang J, Wen TQ, Huang H, Luo D, Ma H, Zhang DB. 2006. The rice tapetum degeneration retardation gene is required for tapetum degradation and anther development. Plant Cell 18, 2999-3014.

Li W, Yang X, Lin Z, Timofejeva L, Xiao R, Makaroff CA, Ma H. 2005. The AtRAD51C gene is required for normal meiotic chromosome synapsis and double-stranded break repair in Arabidopsis. Plant Physiol 138, 965-976.

Liu Z, Bao W, Liang W, Yin J, Zhang D. 2010. Identification of gamyb-4 and analysis of the regulatory role of GAMYB in rice anther development. J Integr Plant Biol 52, 670-678.

Nelson M, Humphrey W, Gursoy A, Dalke A, Kale' L, Skeel RD. 1996. NAMD: A parallel objectoriented molecular dynamics program. Int. J. Supercomput. Appl. High Perform. Comput. 10, 251256.

Niu N, Liang W, Yang X, Jin W, Wilson ZA, Hu J, Zhang D. 2013. EAT1 promotes tapetal cell death by regulating aspartic proteases during male reproductive development in rice. Nat Commun 4, 1445.

O'Malley RC, Huang SC, Song L, Lewsey MG, Bartlett A, Nery JR, Galli M, Gallavotti A, Ecker JR. 2016. Cistrome and epicistrome features shape the regulatory DNA landscape. Cell 165, 12801292.

Ogata K, Morikawa S, Nakamura H, Sekikawa A, Inoue T, Kanai H, Sarai A, Ishii S, Nishimura Y. 1994. Solution structure of a specific DNA complex of the Myb DNA-binding domain with cooperative recognition helices. Cell 79, 639-648.

Pettersen EF, Goddard TD, Huang CC, Couch GS, Greenblatt DM, Meng EC, Ferrin TE. 2004. UCSF Chimera--a visualization system for exploratory research and analysis. J Comput Chem 25, 1605-1612.

Ranjan R, Khurana R, Malik N, Badoni S, Parida SK, Kapoor S, Tyagi AK. 2017. bHLH142 regulates various metabolic pathway-related genes to affect pollen development and anther dehiscence in rice. Sci Rep 7, 43397.

Shinohara A, Ogawa H, Ogawa T. 1992. Rad51 protein involved in repair and recombination in S. cerevisiae is a RecA-like protein. Cell 69, 457-470. 
Sullivan AM, Arsovski AA, Lempe J, Bubb KL, Weirauch MT, Sabo PJ, Sandstrom R, Thurman RE, Neph S, Reynolds AP, Stergachis AB, Vernot B, Johnson AK, Haugen E, Sullivan ST, Thompson A, Neri FV, 3rd, Weaver M, Diegel M, Mnaimneh S, Yang A, Hughes TR, Nemhauser JL, Queitsch C, Stamatoyannopoulos JA. 2014. Mapping and dynamics of regulatory DNA and transcription factor networks in A. thaliana. Cell Rep 8, 2015-2030.

Tsuchiya T, Toriyama K, Ejiri S, Hinata K. 1994. Molecular characterization of rice genes specifically expressed in the anther tapetum. Plant Mol Biol 26, 1737-1746.

van Dijk M, Bonvin AM. 2009. 3D-DART: a DNA structure modelling server. Nucleic Acids Res 37 , W235-239.

Wang Y, Lin YC, So J, Du Y, Lo C. 2013. Conserved metabolic steps for sporopollenin precursor formation in tobacco and rice. Physiol Plant 149, 13-24.

Wei K, Chen H. 2018. Comparative functional genomics analysis of bHLH gene family in rice, maize and wheat. BMC plant biology 18, 309.

Weirauch MT, Yang A, Albu M, Cote AG, Montenegro-Montero A, Drewe P, Najafabadi HS, Lambert SA, Mann I, Cook K, Zheng H, Goity A, van Bakel H, Lozano JC, Galli M, Lewsey MG, Huang E, Mukherjee T, Chen X, Reece-Hoyes JS, Govindarajan S, Shaulsky G, Walhout AJM, Bouget FY, Ratsch G, Larrondo LF, Ecker JR, Hughes TR. 2014. Determination and inference of eukaryotic transcription factor sequence specificity. Cell 158, 1431-1443.

Xiong SX, Lu JY, Lou Y, Teng XD, Gu JN, Zhang C, Shi QS, Yang ZN, Zhu J. 2016. The transcription factors MS188 and AMS form a complex to activate the expression of CYP703A2 for sporopollenin biosynthesis in Arabidopsis thaliana. Plant J 88, 936-946.

Zhang D, Liang W, Yin C, Zong J, Gu F, Zhang D. 2010. OsC6, encoding a lipid transfer protein, is required for postmeiotic anther development in rice. Plant Physiol 154, 149-162.

Zou T, Xiao Q, Li W, Luo T, Yuan G, He Z, Liu M, Li Q, Xu P, Zhu J, Liang Y, Deng Q, Wang S, Zheng A, Wang L, Li P, Li S. 2017. OsLAP6/OsPKS1, an orthologue of Arabidopsis PKSA/LAP6, is critical for proper pollen exine formation. Rice (N Y) 10, 53. 


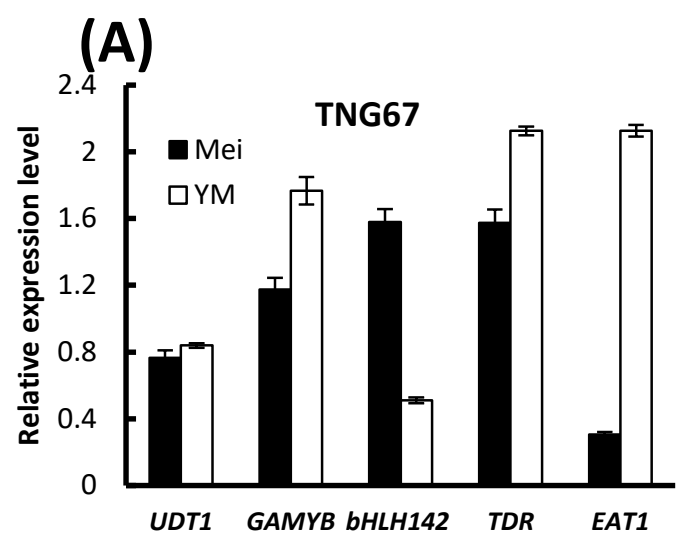

(B)

(C)
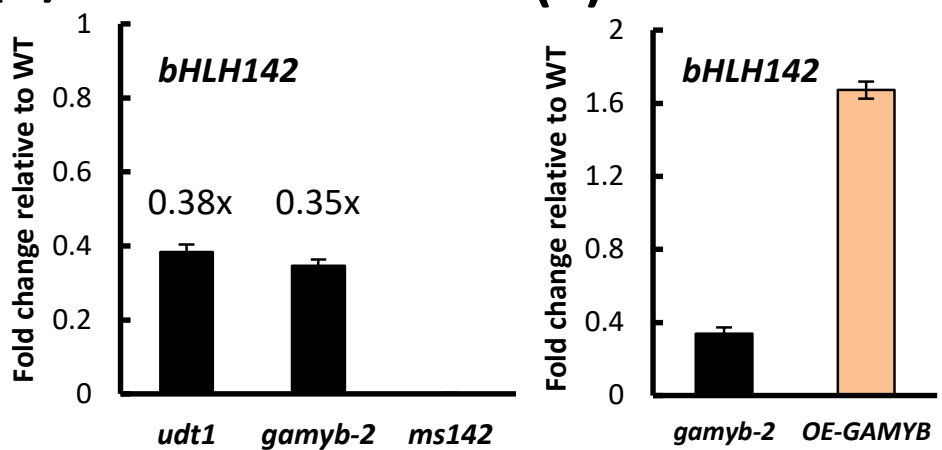

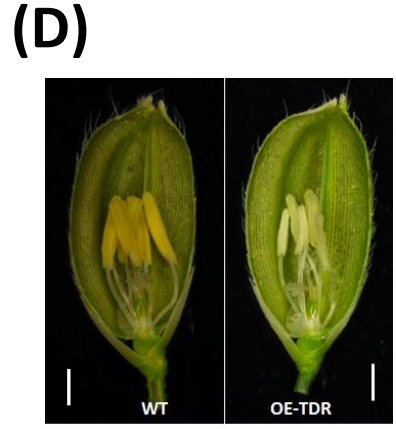

$(\mathbf{F})_{1}$
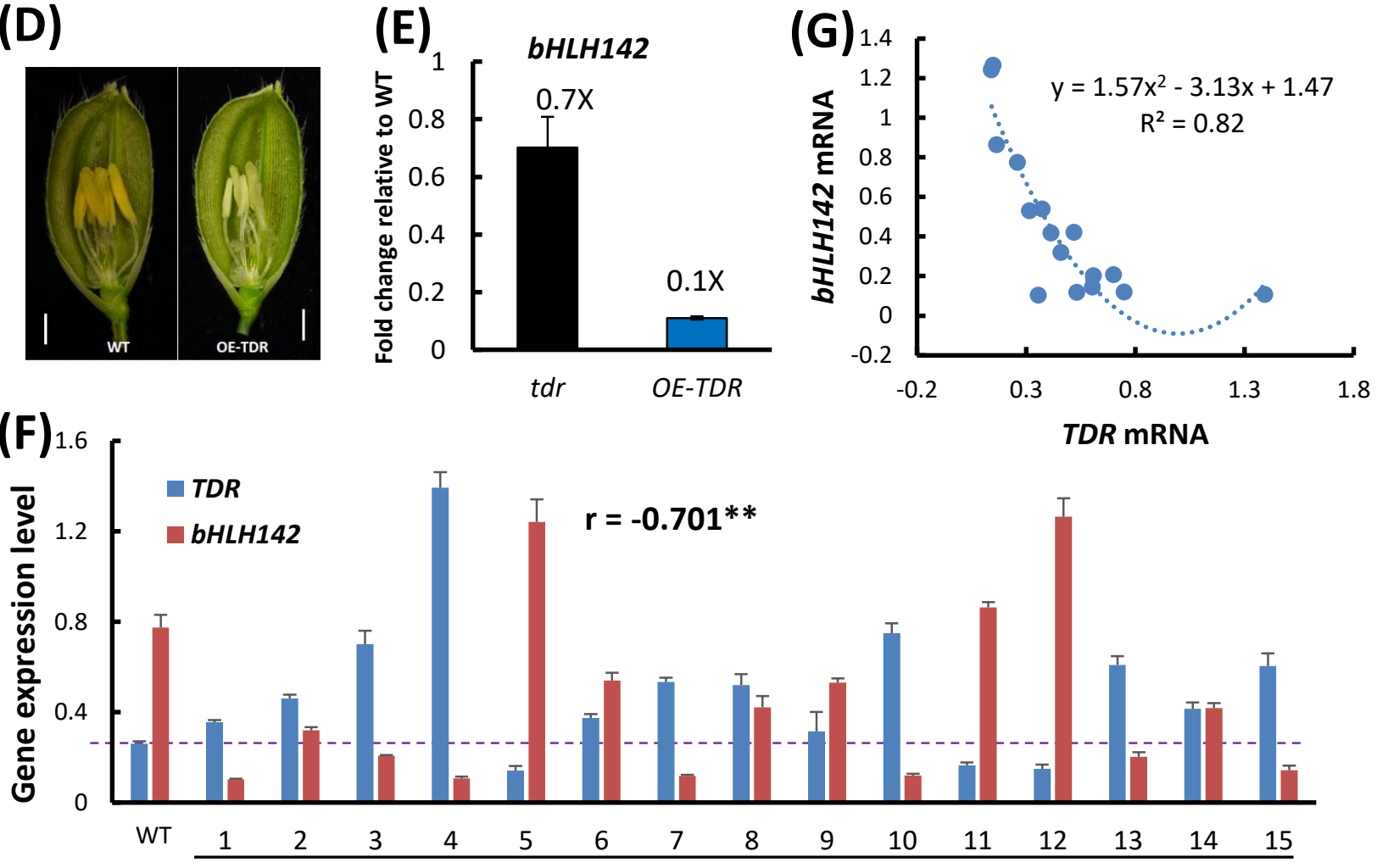

OE-TDR transgenic lines

Fig. 1. Expression patterns of $b H L H 142$ and expression relationship between $b H L H 142$ and TDR. (A) Expression patterns of UDT1, GAMYB, bHLH142, TDR, and EAT1 in anthers of TNG67 at the meiosis (Mei) and young microspore (YM) stages. (B) Expression patterns of bHLH142 in the anthers of $u d t 1$, gamyb-2, and ms142 mutants at the meiosis stage. (C) Down- and up-regulation of bHLH142 in gamyb-2 and OE-GAMYB (line \#6) anthers, respectively. (D) Comparison of the spikelet morphology between TNG67 (WT) and OE-TDR \#4 transgenic rice. The photo was taken one day before anthesis. Bar $=1 \mathrm{~mm}$. (E) Expression patterns of $b H L H 142$ in the anthers of $t d r$ mutant (in Dongjin background) and OE-TDR (in TNG67 background) at the meiosis stage. qRT-PCR data are normalized with respective to WT. (F) Expression patterns of TDR and $b H L H 142$ in OE-TDR transgenic lines. RNA of anthers at the meiosis stage was collected from 15 independent OE-TDR transgenic lines. (G) Relationship between $b H L H 142$ and TDR mRNA levels in TNG67 (WT) and 15 OE-TDR transgenic lines. Regression analysis fit to a binomial regression equation of $y=1.57 x^{2}-$ $3.13 x+1.47\left(R^{2}=0.82\right) ; n=16$ lines. Gene expression levels were normalized to Ubi5 using qRT-PCR. Error bars indicate the SD among three technical replicates. Mei, meiosis; VP, vacuolated pollen. Each experiment was repeated three times. 
(A)

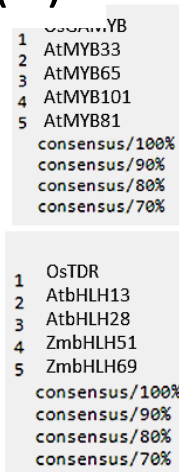

$\begin{array}{rrr}\text { cov } & \text { pid } & 1 \\ 100.0 \% & 100.0 \% & \\ 100.0 \% & 81.6 \% & \\ 100.0 \% & 80.4 \% & \\ 100.0 \% & 76.9 \% & \\ 100.0 \% & 70.9 \% & \\ & & \\ & & \\ & & \end{array}$
cov pid 1 $100.0 \% 100.0 \%$ $100.0 \% \quad 33.7 \%$ $100.0 \% \quad 28.7 \%$ $95.9 \% \quad 29.4 \%$ $100.0 \% \quad 25.7 \%$

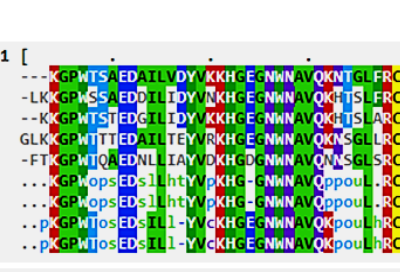

1

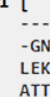

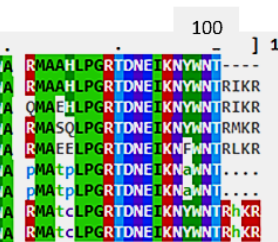

AtMYB33

(Plant Cistrome)

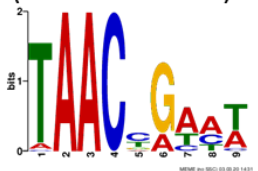

] 87

AtbHLH13 (Cis-BP)

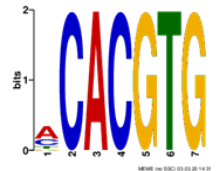

(B)

\section{bHLH142}

os01g0293100 (O. sativa Japonica) LPERR01G11210 (L. perrieri) BRADI_2g11260v3 (B. distachyon) SORBI_3003G140100 (s. bicolor) Zm00001d008174 (z. mays)
$-1010 b$

OsGAMYB
OsTDR

TCAGGTTAA TAACAAACTAATGCCAAAGTTAAAA CAGATA

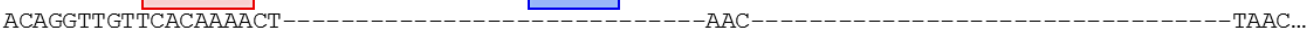
TCAAGCTG---ACTAAATTTATAGAAAAATGTATTAACAACTA-----AATATCAAATAAGTCGTAGATGTTGGTAGAT-TTTTCTAT... GGCTGGTCG-----AAAATTGGAAATAAAATTTACAACTCTTTGTTATTAACAGCAGCTG_AGCGCTTTGCTGGACAATT-TGGCTAA... GCGG--CATqCAACAACCCTCCGGAAGGGTTCAGCCCAACGC------AACCCCGGCCAAACCGCCCACCTCCATGAGTCATTGTTTT...

$-155 b \quad-109 b$

Os01g0293100 LPERR01G11210 BRADI 2g11260v3 SORBI_3003G140100 $\mathrm{Zm} 00001 \mathrm{~d} 008174$
TTTGGAGAGAGGAGGGTCAGTGGGGCCCTCCTGGGAGGAATCCCAGCAACAAACC-ACTOTTCTGATGAAAGGCCAGdCAGCTAAGTGATCT... TTTTGAG---GGGAGGCTGGTGGGGCCCTCCTGGGAGGAATCCCAGCAACAAACC-ACT TTTCTGATGAAAGGCCAGCCGAGCTGCTGGGAT... CFGTAGG---GGGAGGTTAGCGGGGCCCTCCGGGGAGGAATCTCAGTAACAAACC-ACTC-TCTTATGAAAG---AGGC----.---.---... CEAAGTG ------------GGGCCCTCCTGGGAGGAATCTCA TAACAAACOGGCTCTTCCTTCTCAAAGGCAGCCCGGCC--------... TTGGTAGCTAGGCAAGCTAATGGGGTA CATATG GAAGGA-----GGAAACCAAGICGATCGTC------------- GTCGT--

Fig. 2. Inferring DNA-binding motifs of $G A M Y B$ and $T D R$, and identifying their TF binding sites in the promoter sequence of $b H L H 142$. (A) DNA-binding domain (DBD) sequences of GAMYB (top) and TDR (bottom) and their top 4 similar DBDs of TFs with known motifs are shown. The logo of the known motif of AtMYB33 (/AtbHLH13) that has the best similar score with GAMYB (/TDR) is shown at the right panel. The two known motifs (PWMs) are from the Plant Citrome and Cis-BP databases. (B) Alignment of promoter sequences of bHLH142 and four orthologues are shown where two regions in the promoters have been identified as putative TF binding sites of GAMYB and TDR, respectively. The binding site of GAMYB (/TDR) is enclosed by a red (/blue) box. 


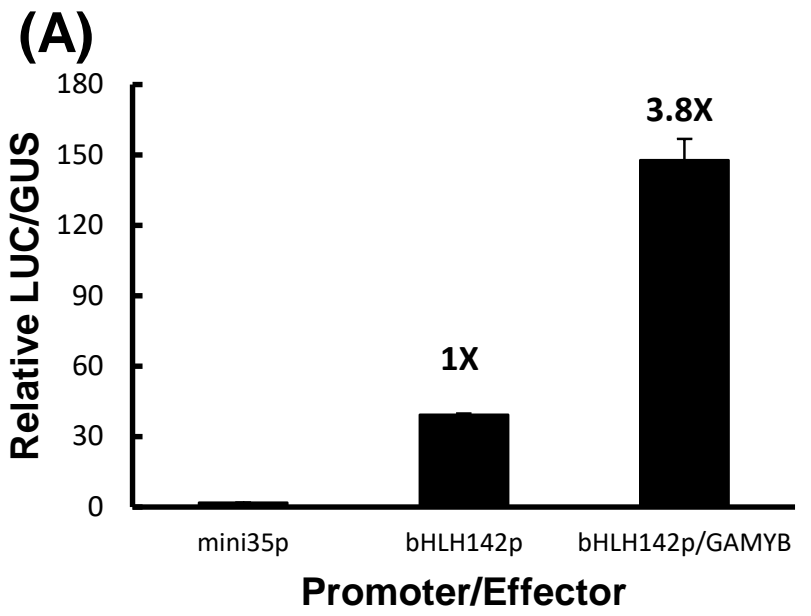

(B)

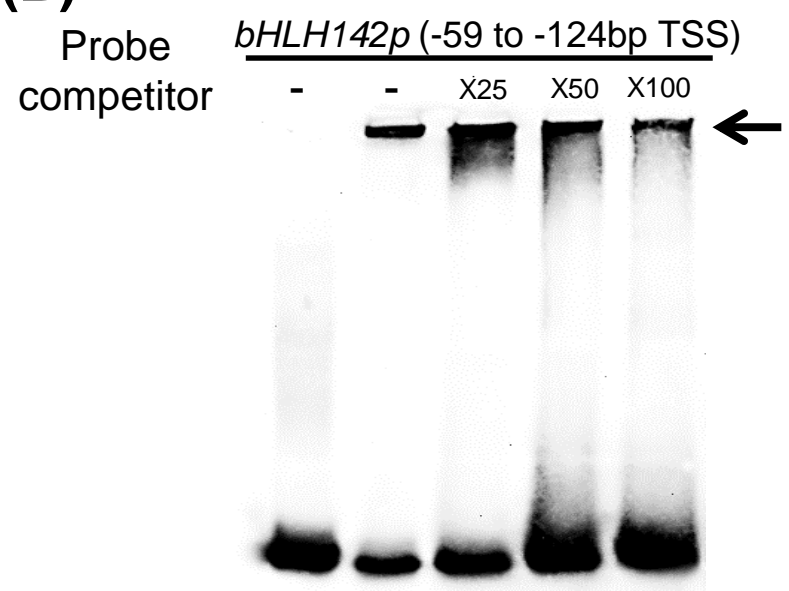

GAMYB protein - ++++

(C)

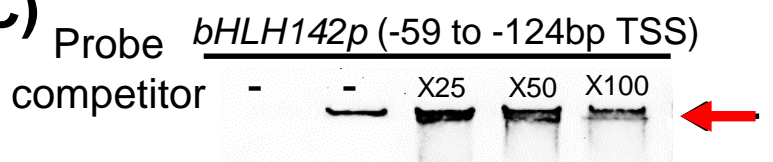

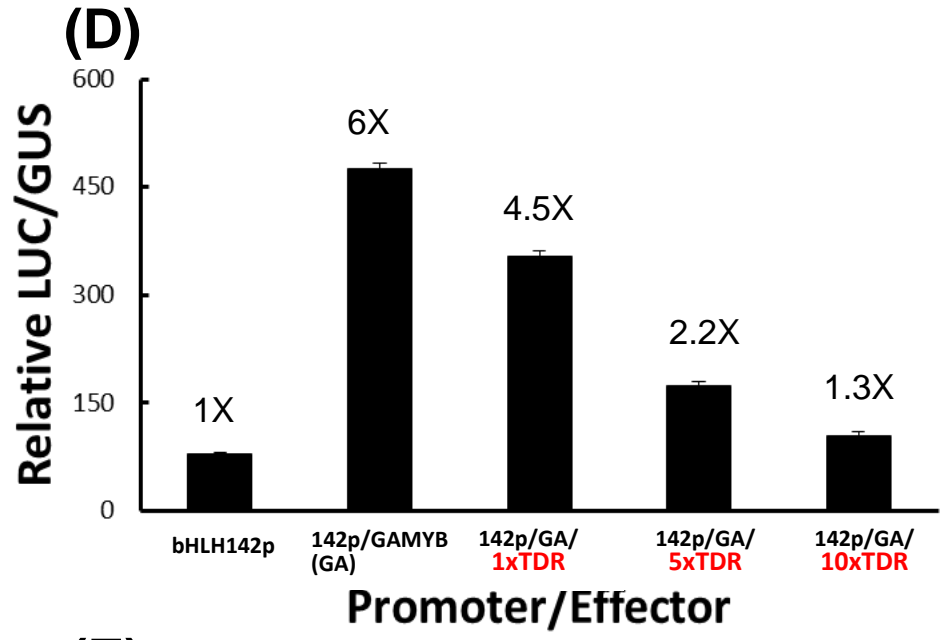

(E)

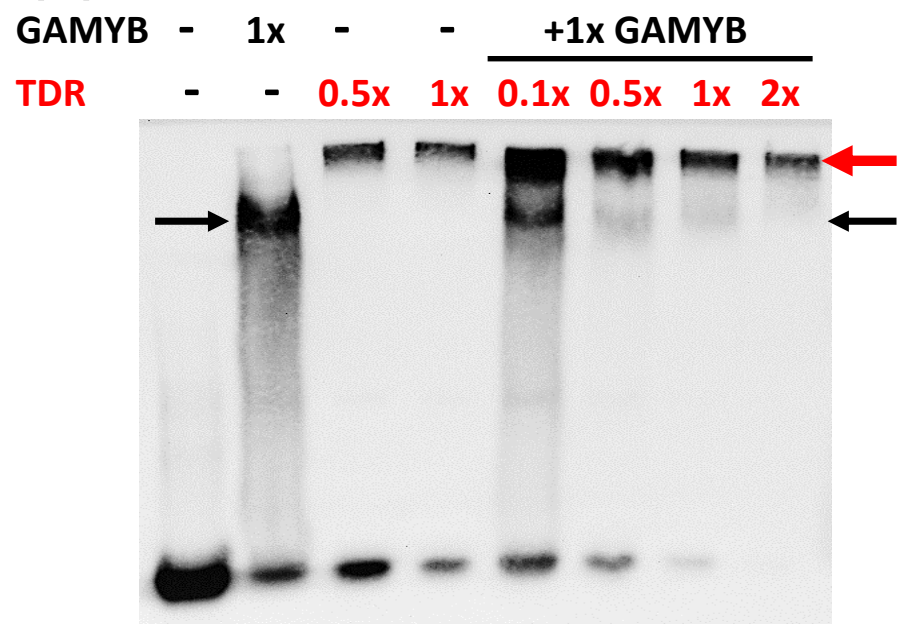

Fig. 3. In vivo and in vitro assays of binding of GAMYB and TDR to bHLH142 promoter. (A) Transactivation of the bHLH142 promoter sequence $(3 \mathrm{~kb})$ fused to the firefly luciferase gene (LUC) reporter gene by GAMYB in rice protoplasts. Different effectors were co-transfected with the reporter and internal control plasmid (pBI221). The data represent means of three independent transient transformations. Transient transformation without the effector plasmid (mini35p) was used as a negative control. Bars indicate the SD of three biological replicates. The experiment was repeated three times. (B) EMSA assay of binding of GAMYB to the promoter region of $b H L H 142$. Probe 1 at -59 to $-124 \mathrm{bp}$ from the transcription start site (TSS) of $b H L H 142$ promoter was synthesized. (C) EMSA assay of binding of TDR to the promoter of $b H L H 142$ probe 1 . The arrows indicate the protein and DNA complex $(B, C)$. The EMSA experiments were repeated three times. (D) Promoter transient assay indicated that TDR protein inhibited GAMYB modulation on bHLH142 promoter-LUC activity. Different molar ratios of TDR (1x, 5x, and $10 \mathrm{x}$ ) and GAMYB proteins were used for transient assays. The experiment was repeated three times. Bars indicate the SD of three biological replicates. (E) EMSA data indicated that TDR inhibited GAMYB binding on $b H L H 142$ promoter probe 1 . The black and red arrows indicated GAMYB/ DNA complex and TDR/ DNA complex, respectively. 

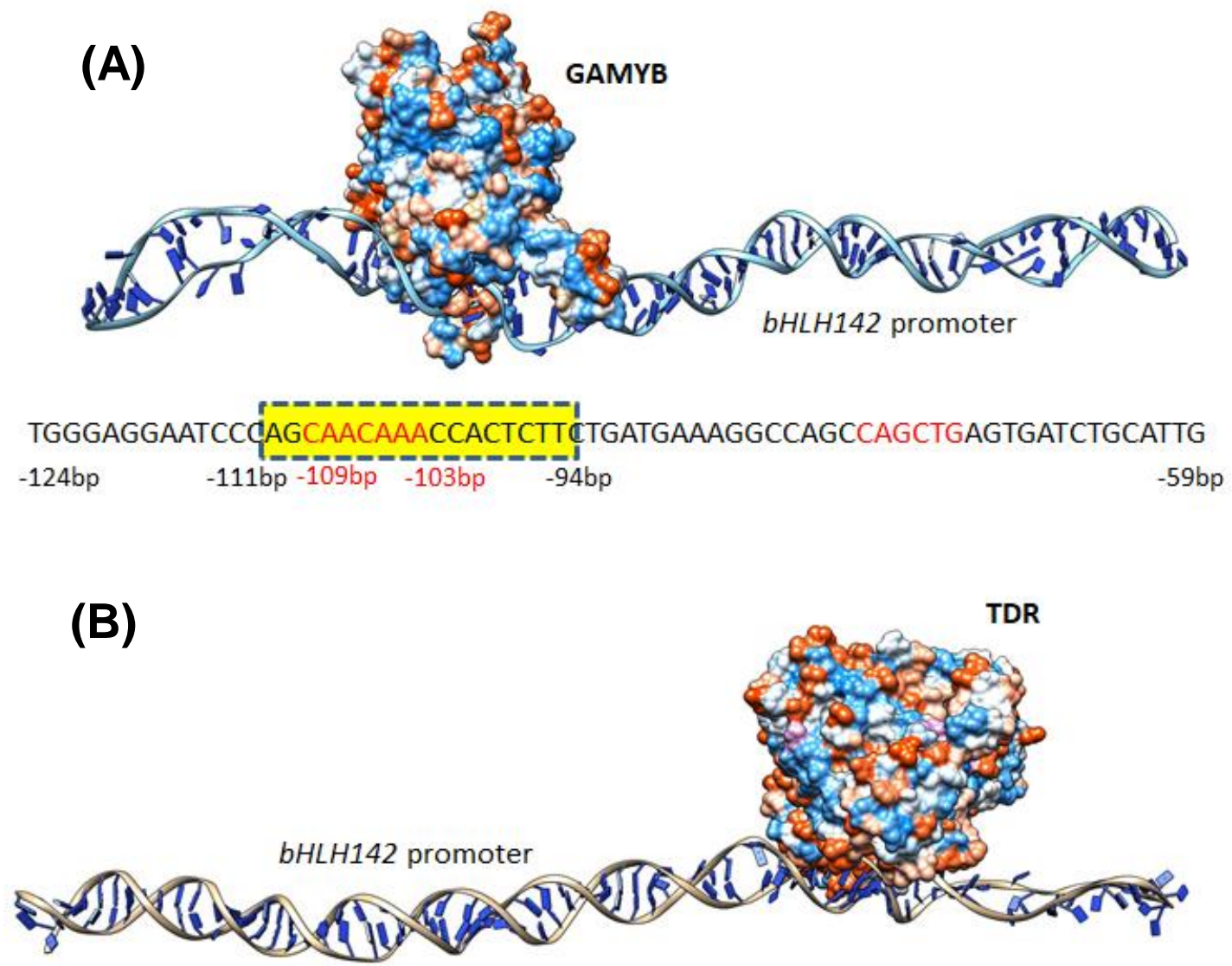

$\begin{array}{lccc}\text { TGGGAGGAATCCCAGCAACAAACCACTCTTCTGATGAAAGGCCAGCCAGCTGAGTGATCTGCATTG } \\ -124 \mathrm{bp} & -78 \mathrm{bp} & -73 \mathrm{bp} & -59 \mathrm{bp}\end{array}$

(C)

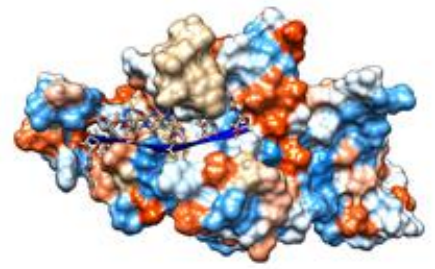

GAMYB interact with CAACAAA Binding Energy: $-170 \pm 57 \mathrm{kcal} / \mathrm{mol}$
(D)

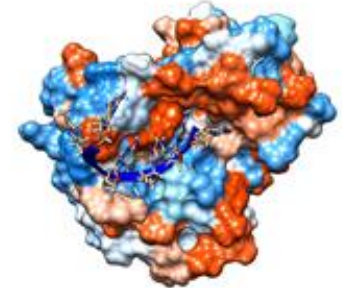

TDR interact with CAGCTG Binding Energy: $-319 \pm 54 \mathrm{kcal} / \mathrm{mol}$

Fig. 4. Molecular dynamics simulations of protein/DNA interaction between GAMYB monomer or TDR homodimer and $b H L H 142$ DNA. (A) Binding of the predicted amino acid patches on GAMYB to the $b H L H 142$ promoter sequence AGCAACAAACCACTCTTC (-94 to -111 bp from TSS). (B) Binding of the predicted amino acid patches on TDR homodimer to the $b H L H 142$ promoter sequence CAGCTG (-73 to -78 bp from TSS). (C) Interaction between GAMYB and the CAACAAA motif with a binding energy of $-170 \pm 57 \mathrm{kcal} / \mathrm{mol}$. (D) Interaction of TDR with an E-box (CAGCTG) with a binding energy of $-319 \pm 54 \mathrm{kcal} / \mathrm{mol}$. 


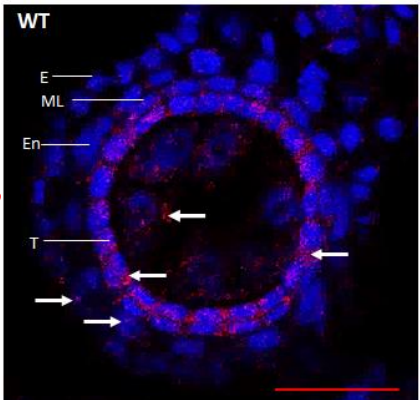

Cy3 $(543 \mathrm{~nm})$ ms142

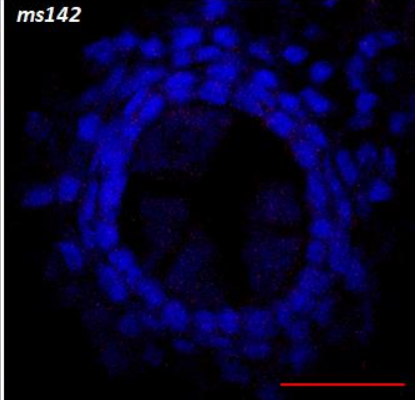

Cy5 $(633 \mathrm{~nm})$

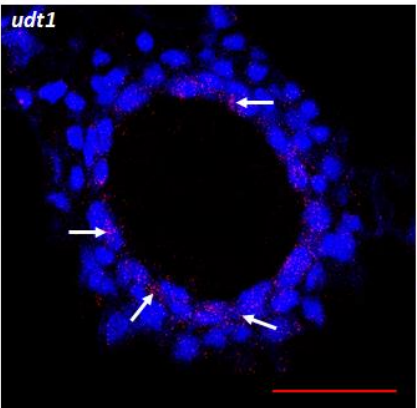

Cy3 + Cy5

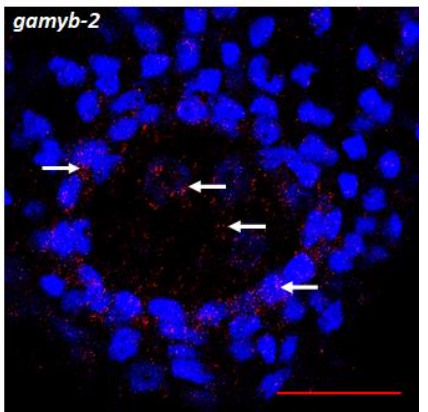

Cy $3+$ Cy5 + BF
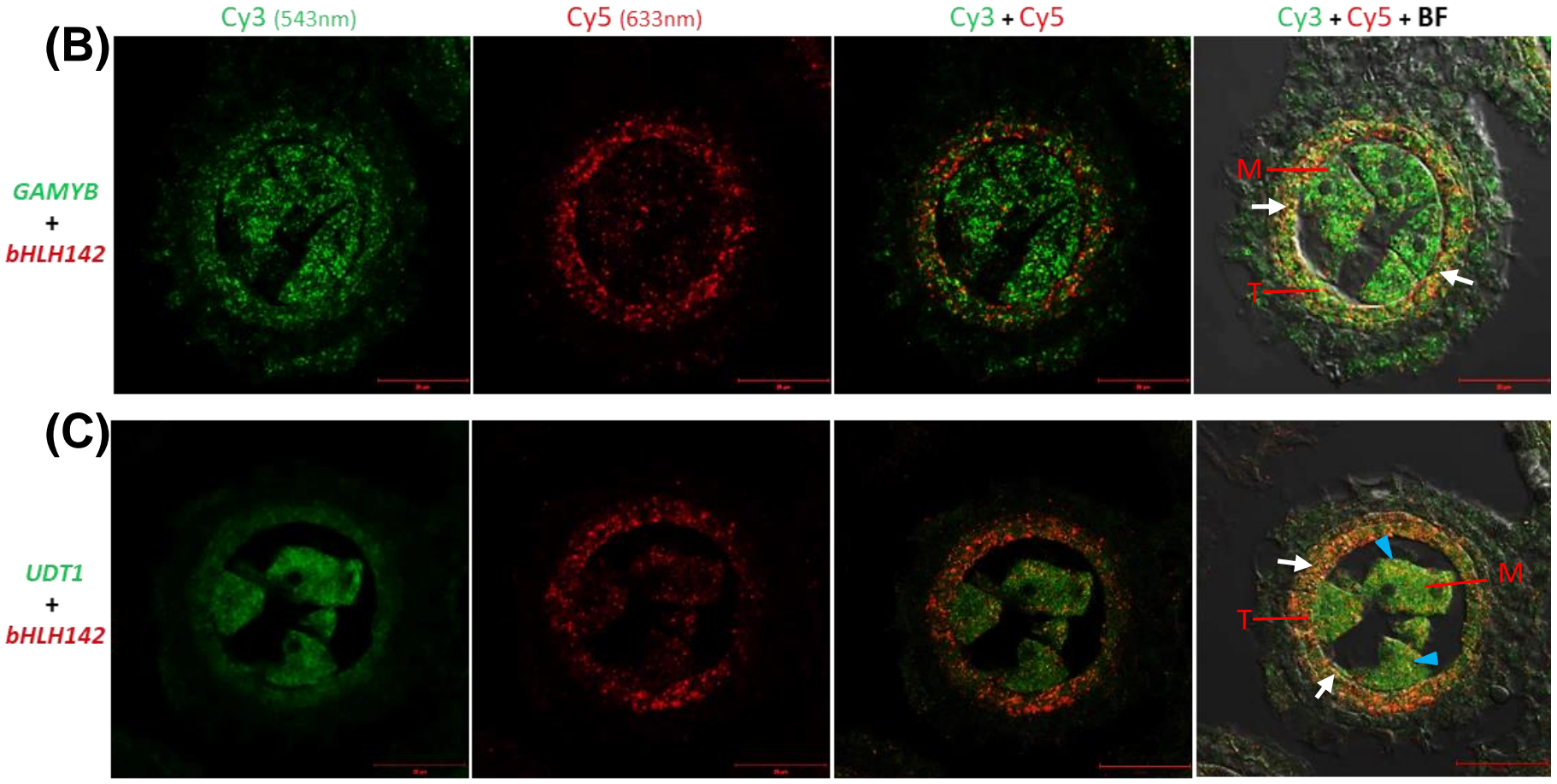

Fig.5. RNA fluorescent in situ hybridization (FISH) study of the expression patterns of $b H L H 142$ in mutants and two-color fluorescent in situ hybridization (DISH) study of the co-expression of UDT1 and GAMYB with bHLH142 in anthers at early meiosis. (A) RNA single color FISH showing the expression pattern of $b H L H 142$ in the anthers of WT, ms142, udt1 and gamyb-2 mutants during early meiosis. Anther tissues were stained with TSA working solution containing Cyanine 5 Plus Amplification Reagent (300x dilution). Ex/Em: 633/670 nm. The arrow indicates the FISH signal (red color). Tissues were counter stained with DAPI (4',6-diamidino-2-phenylindole). E, epidermis; En, endothecium; ML, middle layer; $T$, tapetum. Bars $=20 \mu \mathrm{m}$. (B) DISH showing coexpression of GAMYB and $b H L H 142$ in the tapetal layer of the anther. Green and red spots show fluorescent signals of GAMYB and bHLH142, respectively. Yellow spots show the co-expression of GAMYB and $b H L H 142$ mRNAs in the tapetal cells (white arrows). (C) DISH showing co-expression of UDT1 and bHLH142 in the tapetal layer and meiocytes of the anther. Green and red spots show fluorescent signals of UDT1 and $b H L H 142$, respectively Yellow spots show coexpression of UDT1 and $b H L H 142$ in the tapetal cells (white arrows) and meiocytes (blue arrow heads). Pictures were taken under $100 \mathrm{X}$ objective oil immersion lens. $\mathrm{T}$, tapetal layer; $\mathrm{M}$, meiocytes. Bars $=20 \mu \mathrm{m}$. 


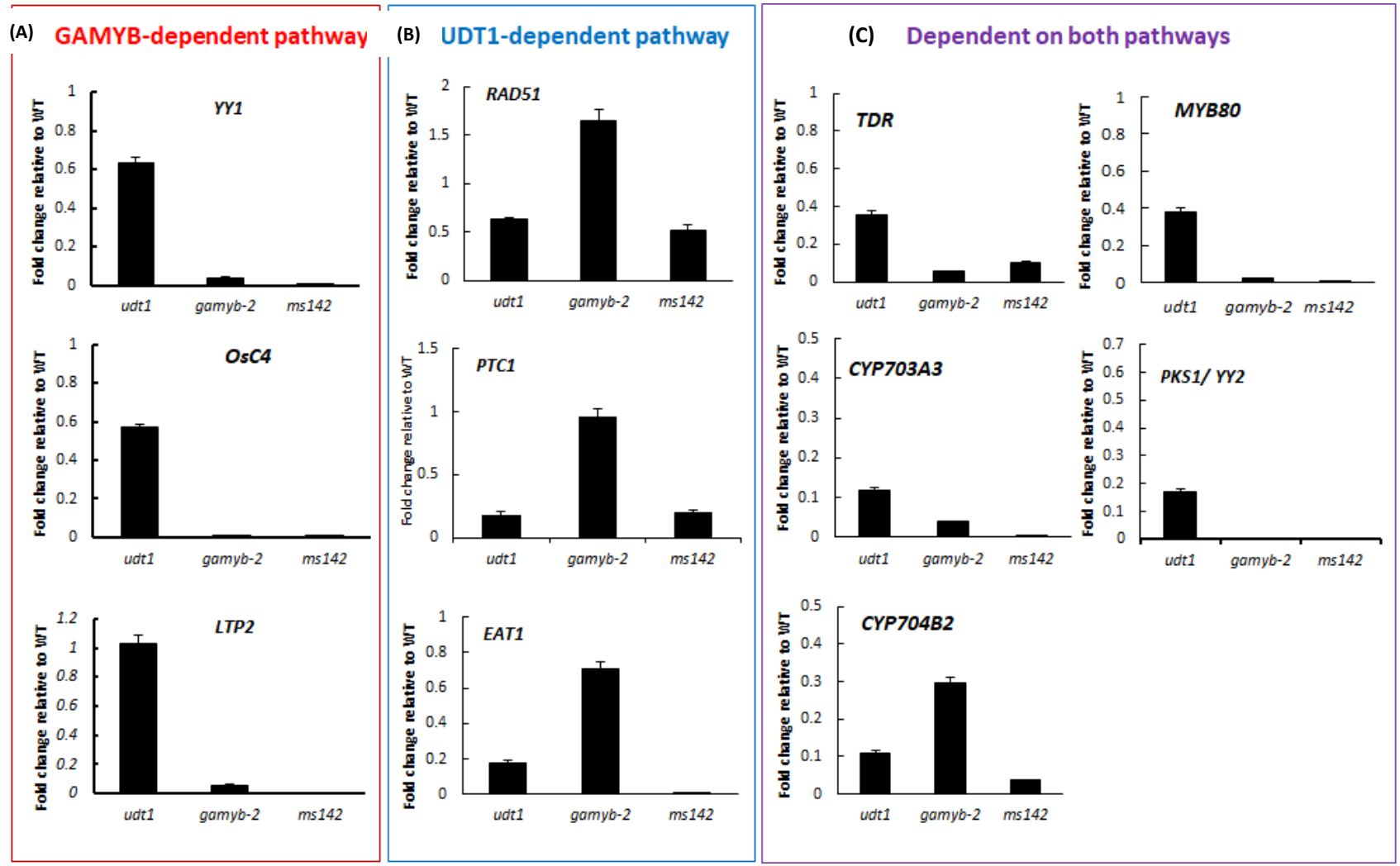

Fig. 6. Dependence of the expression of pollen marker genes on GAMYB, UDT1, or both pathways. Expression patterns of some pollen marker genes in the anthers of udt1, gamyb-2, and ms142 mutant lines were studied using qRT-PCR. (A) YY1, OsC4, and LTP2 were found dependent on GAMYB- and bHLH142-regulatory pathways. (B) RAD51, PTC1, and EAT1 were dependent on UDT1- and bHLH142-regulatory pathways. (C) TDR, CYP703A3, Cyp704B2, MYB80, and PKS1/YY2 were dependent on both pathways. Gene expression levels were normalized to Ubi5, and the fold change relative to the respective Wild-type is presented. Error bars indicate the SD of three technical replicates. Each experiment was repeated three times. 


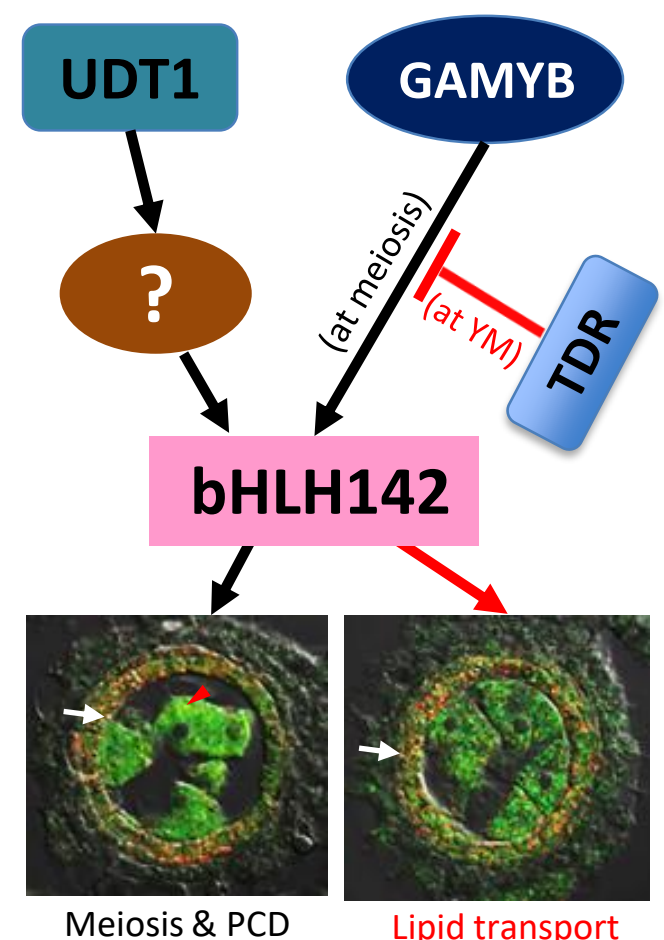

Fig. 7. Model of this study. bHLH142 acts downstream of UDT1 and GAMYB and works as a "hub" in these two pollen pathways. GAMYB can directly modulate the transactivation of the $b H L H 142$ promoter, but UDT1 cannot. GAMYB modulates $b H L H 142$ at the early stage of meiosis, but the modulation is repressed by TDR at the young microspore stage to keep the homeostasis of $b H L H 142$ gene expression to ensure normal pollen development. Our FISH and DISH data reveals that GAMYB modulates $b H L H 142$ in the tapetal layer and GAMYB-dependent pathway functions in lipid transport. However, the UDT1-dependent pathway regulates $b H L H 142$ in the tapetal layer and meiocytes and plays several other roles in meiosis and tapetal programmed cell death. YM, young microspore stage; $M$, meiocytes; $T$, tapetum; $P C D$, programmed cell death. 